\author{
James E. Chisum \\ Young S. Shin \\ Mechanical Engineering Department \\ Naval Postgraduate School \\ Monterey, CA 93943-5107
}

\section{Explosion Gas Bubbles Near Simple Boundaries}

Finite element analyses of explosion gas bubbles show that including the compressibility of the surrounding media leads to appreciable differences in key areas of the bubble's behavior. In order to more fully understand the behavior of bubbles created by detonations near simple boundaries, analyses incorporating fluid compressibility were conducted at various stand-off distances from simple rigid and constant pressure surface boundaries. The results from these analyses serve to characterize the behavior of the bubbles for the charge type, charge weight, and hydrostatic pressure used in the analyses. (c) 1997 John Wiley \& Sons, Inc.

\section{INTRODUCTION}

When an explosive is detonated underwater, the explosive material is very rapidly converted into gaseous reaction products, which in most cases maintain a closed, simply connected topology for a relatively long period of time. The ability of this explosion gas bubble to contribute significantly to damage to nearby marine structures has been recognized for some time; much of the pioneering research into explosion gas bubbles dates back to the second world war. Many of the more important papers from this time can be found in a collection published by the Office of Naval Research, Department of the Navy (Hartmann and Hill, 1950).

The propagation of the detonation wave front through an explosive is so rapid that the gas products directly behind this wave front are not in pressure equilibrium with the gases further behind the wave front. The gases behind the detonation wave front in fact have a pressure profile similar to a step increase followed by an exponential type decay, with the highest pressure immediately behind the detonation wave front and the decay length increasing with propagation distance. As the deto- nation wave front reaches the boundary between the explosive and the surrounding fluid, a high pressure step-exponential type shock wave is transmitted to and propagates through this fluid. An appreciable fraction of the total explosion gas product energy is lost to the surrounding fluid through radiation of this initial shock wave. However, about half of the initial internal energy of the explosive remains in the gas bubble (Cole, 1948). In a relatively short period of time (the remaining phenomena described take place on a much longer time scale than completion of detonation and radiation of the initial shock wave) the explosion gas products are nearly in pressure equilibrium, with a pressure significantly higher than the hydrodynamic pressure in the surrounding fluid. The gas bubble thus begins expanding against the surrounding fluid. (This expansion begins as soon as the detonation front reaches the explosive boundary, but the time scale allows us to imagine the bubble gases as being homogeneous at the start of this expansion.) The initial pressure within the bubble and the outward velocity of the bubble boundary are relatively large and the surrounding water is relatively easily displaced, so

Received December 1, 1995; Accepted September 4, 1996. Shock and Vibration, Vol. 4, No. 1, pp. 11-25 (1997) (c) 1997 by John Wiley \& Sons, Inc. 
the bubble continues expanding beyond the point at which its internal pressure would be equal to the hydrostatic pressure in the surrounding fluid. This pressure difference between the bubble pressure and the pressure in the surrounding fluid when the bubble boundary velocity reaches zero thus causes the bubble to begin contracting.

Just as the initial conditions (bubble's initial pressure and boundary velocity and momentum field of surrounding fluid) causes the bubble to expand well beyond the point at which it would be in pressure equilibrium with the fluid media, the new "initial" conditions in the bubble gases and the surrounding fluid when the bubble reaches its maximum expansion (bubble boundary velocity equal to zero) causes the bubble to begin contracting and to continue contracting beyond the point at which its internal pressure would equal the hydrostatic pressure in the fluid. Eventually the pressure in the gas bubble is great enough that the momentum field in the surrounding fluid cannot cause further contraction; the bubble is compressed by more remote fluid. This is only significant when the bubble is near maximum volume, but this secondary pressure pulse phenomena leads to an energy loss of about one-third of the remaining bubble energy (Cole, 1948). When the bubble reaches its resultant minimum volume, the pressure in the bubble is again significantly higher than hydrostatic pressure in the surrounding fluid and the stage is set for another expansion-contraction cycle. As many as 10 such cycles have been observed (Cole, 1948).

Additional phenomena occur when an underwater explosion takes place near a boundary. Near a rigid boundary, the fluid on the side of the bubble near the boundary when the bubble is expanding from its minimum volume is not displaced as easily as fluid away from the boundary, leading to movement away from the boundary. However, the bubble rapidly expands to the point where its internal pressure is less than hydrostatic, and only a small displacement away from the rigid boundary occurs during this period. When the bubble pressure falls below hydrostatic pressure, the fluid remote from the rigid boundary more readily accelerates toward the bubble boundary. The bubble thus moves closer to the wall. Because the bubble spends most of its time in this state, significant momentum is imparted to a large volume of fluid when the bubble is large. As the bubble then begins contracting, this fluid momentum becomes concentrated in a small amount of fluid near the bubble, and as the bubble contracts it is accelerated toward the wall.
Because the bubble spends most of its time in the "below hydrostatic pressure" condition, the motion toward the wall gently exceeds the initial motion away from the wall (Cole, 1948). The opposite takes place for a free surface: the fluid momentum field dictates that the bubble initially move toward the surface, but then move rapidly away from the surface. In this article we quantitatively examine the effects of plane rigid wall and constant pressure boundaries on an explosion gas bubble, using the finite element method. An Euclerian finite element mesh, in which the finite element mesh is stationary and material flows through the mesh, was utilized. The finite element program MSC/DYTRAN (MacNeal-Schwendler Corporation, 1995) was used for these analyses.

\section{NUMERICAL APPROACH}

The finite element program MSC/DYTRAN (MacNeal-Schwendler Corporation, 1995) has both Lagrangian and Eulerian processors; only the hydrodynamic multimaterial Eulerian processor has been used in the analyses described in this article. This Eulerian processor uses the basic conservation equations, in conjunction with constitutive equations and a material transport scheme, to compute the solution in space.

The control volume method is used, with each element as a separate control volume. The basic conservation equations for a hydrodynamic material expressed in the control volume formulation are

$$
\begin{gathered}
\frac{\partial}{\partial t} \iiint_{\mathrm{vol}} \rho d V=-\iint_{\mathrm{surf}} \rho(\mathbf{u} \cdot d \mathbf{S}), \\
\frac{\partial}{\partial t} \iiint_{\mathrm{vol}} \rho \mathbf{u} d V=-\iint_{\mathrm{surf}} \rho \mathbf{u}(\mathbf{u} \cdot d \mathbf{S}), \\
\frac{\partial}{\partial t} \iiint_{\mathrm{vol}} \rho e d V=-\iint_{\mathrm{surf}} \rho e(\mathbf{u} \cdot d \mathbf{S}),
\end{gathered}
$$

for conservation of mass, momentum, and energy, respectively. A one point approximation (the value of each property at the geometric center of each element) is used to calculate these integrals, in conjunction with the interpolated velocity values at the faces between adjacent element.

The material transport scheme used in this program is a first-order "donor-acceptor" scheme in which transported quantities are subtracted from donor elements and added to acceptor elements based upon donor element values and the interpo- 
lated velocity at the common element face. Thus, for transport from element $m$ to element $n$,

$$
\mathbf{u}_{\mathrm{face}}=\frac{1}{2}\left(\mathbf{u}_{m}+\mathbf{u}_{n}\right)
$$

and during time step $d t$ the volume transport is

$$
d V=\mathbf{u}_{\mathrm{face}} \cdot d \mathbf{S} d t
$$

The mass, momentum, and energy transport from element $m$ to element $n$ during this time step is then

$$
\begin{gathered}
d M=\rho_{m} d V, \\
d(\mathbf{M})=\rho_{m} \mathbf{u}_{m} d V, \\
d E=\rho_{m} e_{m} d V .
\end{gathered}
$$

The solution in time is computed using an explicit central finite difference method; the time step is calculated internally at each time step to satisfy the requirement that

$$
d t=s \frac{d L}{c+u}
$$

where $s$ is the safety factor $(<1), L$ is the smallest characteristic element length, $c$ is the wave velocity, and $u$ is the particle velocity.

\section{PROBLEM DESCRIPTION}

Two different basic problems were analyzed. The geometry of the first problem was chosen to correspond to one of a set of experiments conducted at the Woods Hole Oceanographic Institute during and shortly after the second world war (Swift and Decius, 1950). This experiment measured the maximum bubble radius and the bubble oscillation period resulting from detonation of a small (0.299 kg TNT equivalent including booster) TNT charge located at a depth of $178.6 \mathrm{~m}$ in seawater. Because there was no nearby boundary in this experiment, it approximates the simplest possible case in which the boundary is located at infinity. This problem was used to examine both the adequacy of the state equations used and the reasonableness of the modeling assumptions (described below) in approximating the underlying physics of the deep

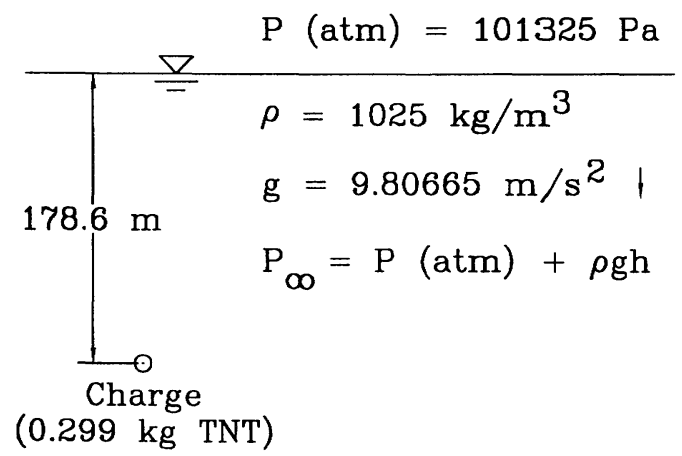

FIGURE 1 Problem geometry for the spherical bubble problem.

explosion problem. The geometry of this essentially free-field problem is illustrated in Fig. 1.

The other problem studied consists of a $10.24 \mathrm{~kg}$ cylindrical TNT charge detonated in seawater at a depth of $1000 \mathrm{~m}$ at various stand-off distances from a plane constant pressure surface or rigid wall boundary, as illustrated in Fig. 2. The charge was assumed to be cylindrical with a diameter to height ratio of one for convenience. Underwater explosion experiments also typically use cylindrical shaped charges, for practical reasons. The detonation initiation point within the charge was assumed to be at the midpoint of the axis of the charge. High-order detonation was assumed to occur without a significant weight of booster charge material.

A number of simplifying modeling assumptions were made for both of these problems. Because experimental results show that very deep charges undergo little migration due to gravity (Cole, 1948), gravity was neglected except for its effect on the hydrostatic pressure at the depth of the charge. All of the fluid surrounding the charge was then assumed to initially have this hydrostatic pressure. The fluid was assumed to be compressible but inviscid and irrotational.

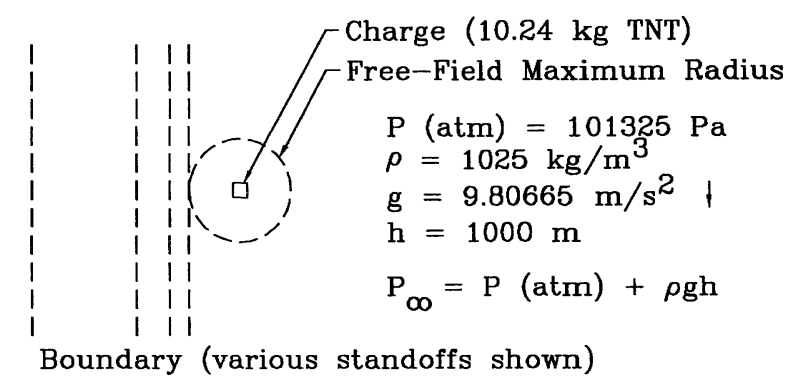

FIGURE 2 Problem geometry for the bubble near the boundary problem. 


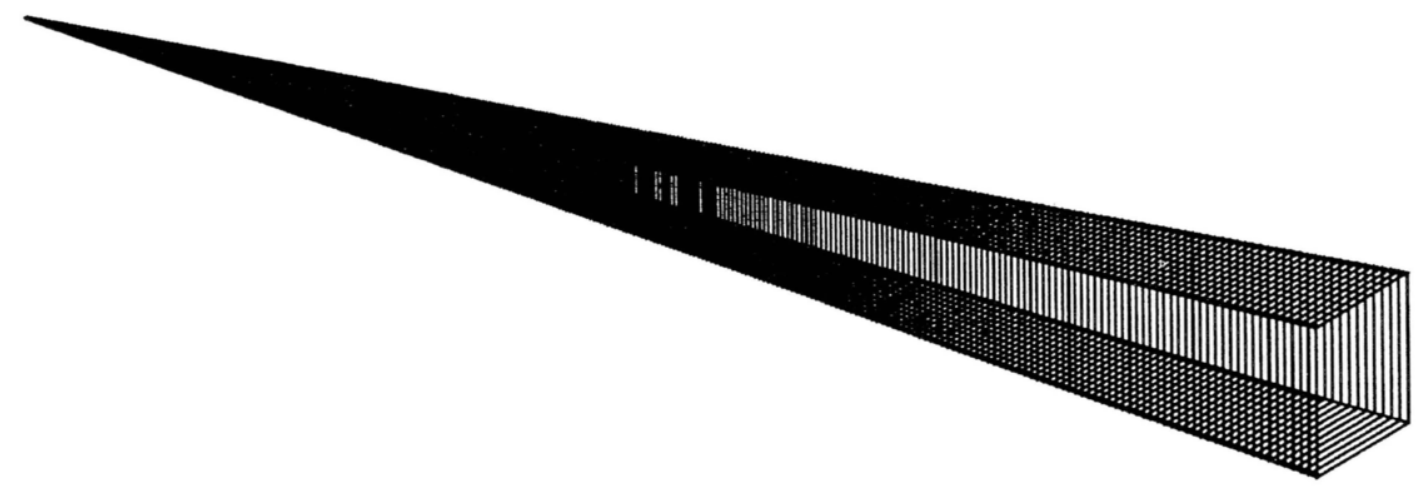

REGION NEAR CHARGE

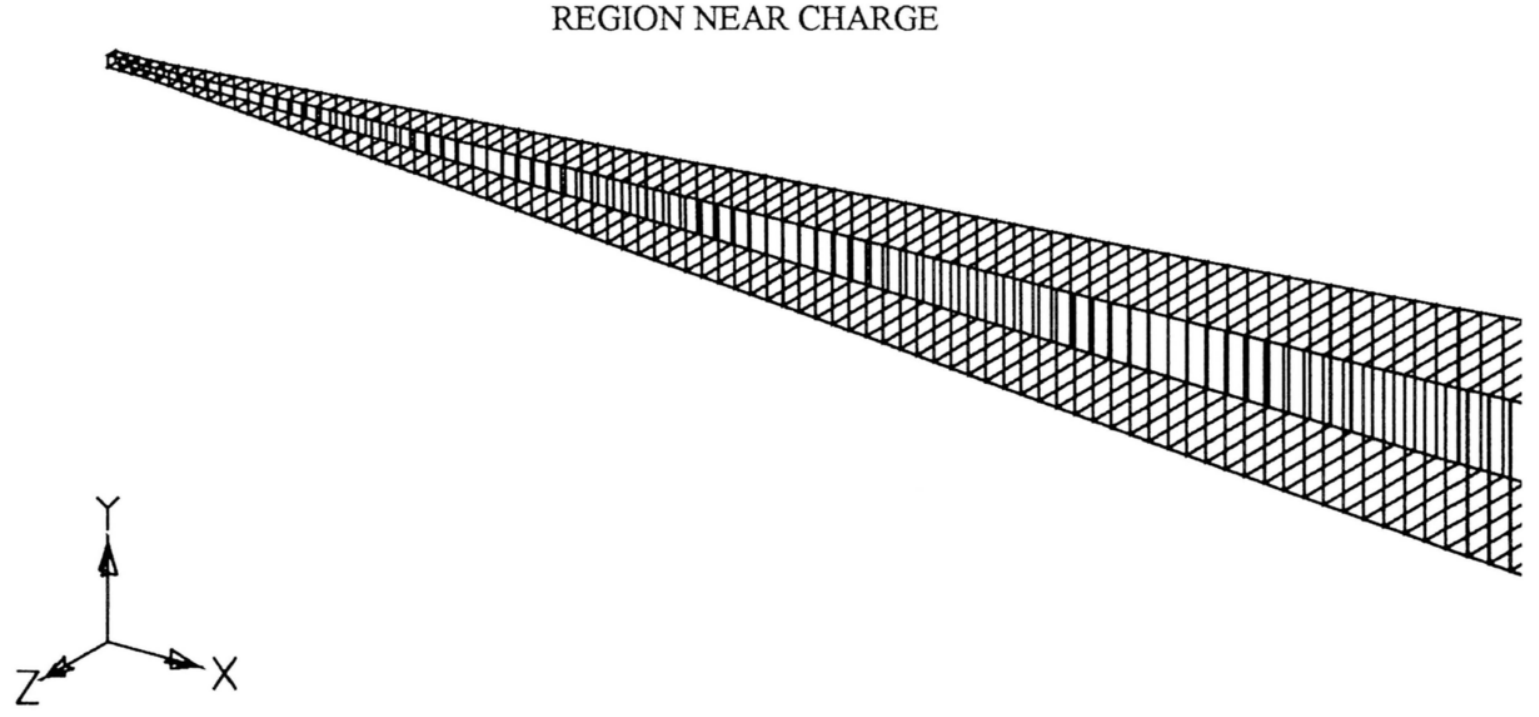

FIGURE 3 Finite element model for the spherical bubble problem.

Heat transfer between the surrounding fluid and the explosion product gases were assumed to be negligible over the time frame of the problem, and as a result vaporization of seawater at the interface between the two was assumed to be insignificant. Mass transfer between the seawater and the explosion product gases, a fraction of which would be water vapor, was also assumed to be negligible.

\section{MODEL DESCRIPTION}

The TNT in these problems was modeled using the Jones-Wilkins-Lee equation of state, with state equation parameters taken from the Lawrence Livermore National Laboratories Explosives
Handbook (Dobratz, 1981). This state equation provides a relationship between the pressure in the explosion product gases behind the detonation front and the density and specific internal energy of these gases. At any moment in time, the pressure within a small region in the explosion product gases is given by

$$
\begin{aligned}
P= & A\left(1-\frac{\omega \eta}{R_{1}}\right) e^{-R_{1} / \eta} \\
& +B\left(1-\frac{\omega \eta}{R_{1}}\right) e^{-R_{2} / \eta}+\omega \eta \rho_{0} E,
\end{aligned}
$$

where $E$ is the specific internal energy per unit mass; $\rho_{0}$ is the reference density; $\rho$ is the overall material density; $\eta$ is the $\rho / \rho_{0}$; and $A, B, \omega, R_{1}$, and 
$R_{2}$ are state equation parameters for the explosive [numerical values given by Dobratz (1981)]. A spherical detonation wave front traveling outward from the initiation point at the center of the charge at a velocity of $6930 \mathrm{~m} / \mathrm{s}$ was used.

The seawater was modeled using a polynomial equation of state; parameters for this state equation were obtained by fitting published data to this state equation form (Chisum and Shin, 1995). This state equation accounts for changes in the pressure of the seawater due to density changes (compressibility) and specific internal energy changes (from work done on or by the seawater). In compression, the seawater pressure is given by

$$
P=a_{1} \mu+a_{2} \mu^{2}+a_{3} \mu^{3}+\left(b_{0}+b_{1} \mu+b_{2} \mu^{2}\right) \rho_{0} E,
$$

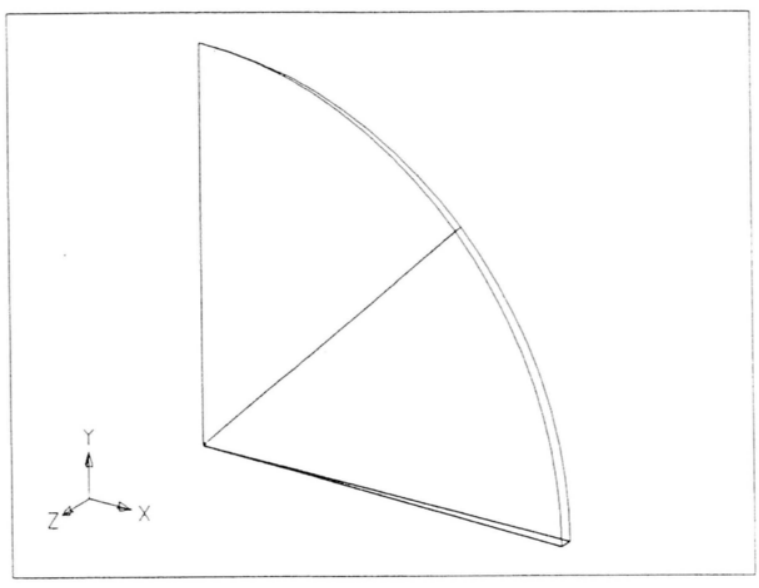

(a)

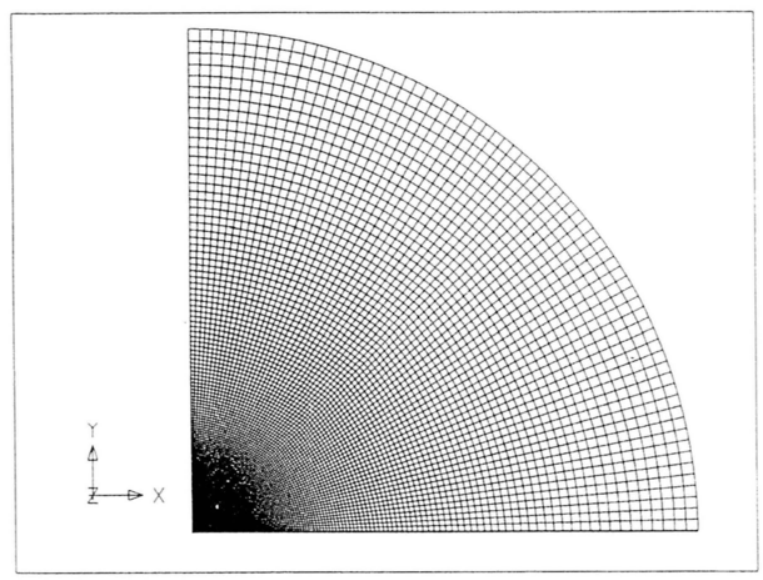

(c) and in tension, the pressure is given by

$$
P=a_{1} \mu+\left(b_{0}+b_{1} \mu\right) \rho_{0} E,
$$

where $\mu=\eta-1$ (the acoustic condensation) and $a_{1}, a_{2}, a_{3}, b_{0}, b_{1}$, and $b_{2}$ are state equation parameters for the seawater [numerical values given by Chisum and Shin (1995)]. A minimum pressure of zero was defined for the seawater, so that if at any time during the transient analyses the flow was such as to give a portion of the seawater a negative total (hydrostatic plus dynamic) pressure, all of the seawater would flow out of that region and a void would be created.

To initialize the pressure in the seawater to the hydrostatic pressure for the two problems analyzed, the specific internal energy rather than the

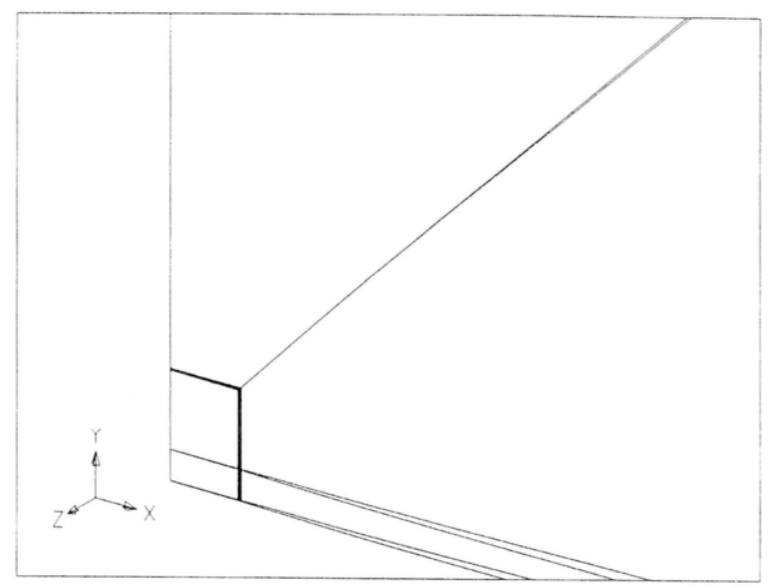

(b)

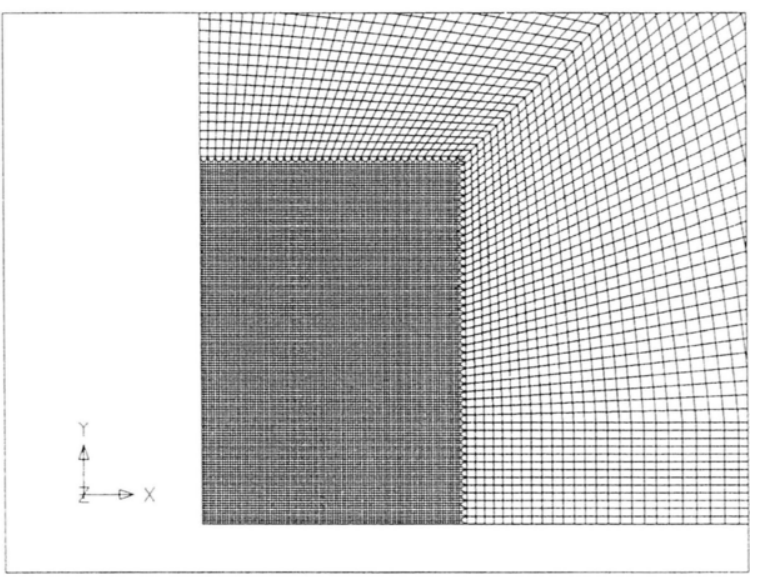

(d)

FIGURE 4 Finite element model for the bubble near the boundary problem (typical). 

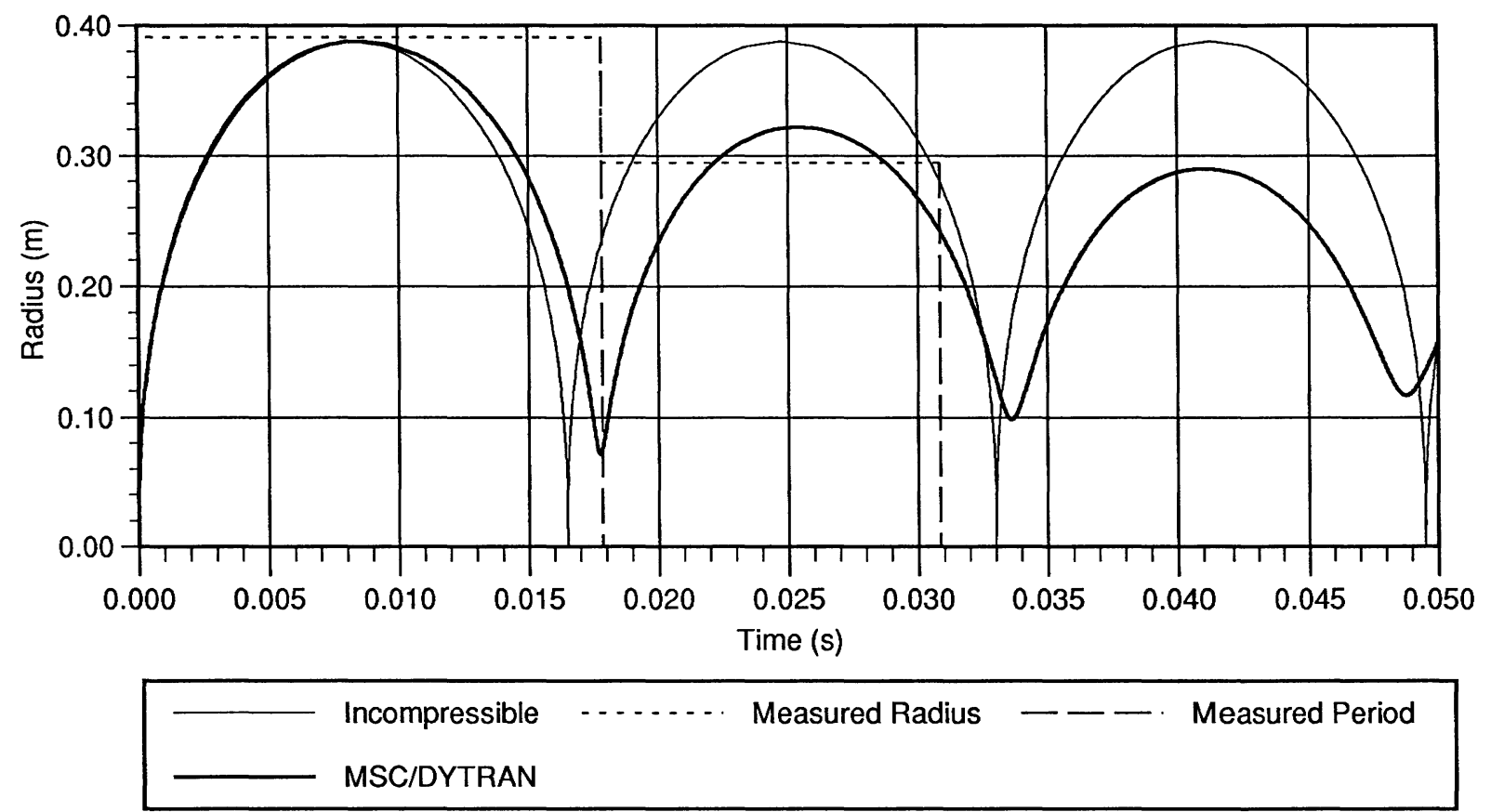

FIGURE 5 Radius versus time behavior for the spherical bubble problem.

acoustic condensation was adjusted. This allows the proper hydrostatic pressure to be set accurately. In these problems, the change in density caused by the seawater head is so small (owing to the relatively incompressible nature of seawater) that significant round-off errors are introduced by using the acoustic condensation to set the hydrostatic pressure.

Because gravity was neglected except for its effect on the hydrostatic pressure in the fluid, which was assumed to be uniform and equal to the hydrostatic pressure at the depth of the charge, the first (Swift and Decius, 1950) problem is essentially spherically symmetric. To model this 1-dimensional problem with the 3-dimensional finite element program MSC/DYTRAN, a tall, thin pyramid shaped volume of fluid (with a $1: 10$ base to height ratio) was used. A very large volume of fluid was modeled to prevent reflection from the boundary from affecting the explosion gas bubble during the time frame of the analysis. Because gradients are small well away from the bubble, a nonuniform mesh spacing was used. Figure 3 shows the overall geometry of the finite element model used for this problem, as well as a close up of the model in the area near the charge. A detailed description of this finite element model is given by Chisum and Shin (1995).

The cylindrical charge in the second problem was placed with its axis normal to the plane boundary. With this orientation, the problem is axially symmetric, with the symmetry axis being the axis of the charge. To model this axially symmetric problem with the 3-dimensional finite element program MSC/DYTRAN, a wedge shaped volume comprising $2^{\circ}$ of arc was modeled. During model development, it was found to be necessary to include a fairly large region of fluid remote from the area of interest near the charge to get acceptable results. This was probably due to the inaccuracies inherent in modeling a "nonreflecting" boundary and to the sensitivity of the bubble motion at the start of the collapse phase to the surrounding flow field, because this is when the flow begins reversing direction. This a very large (400-m radius) volume of fluid was modeled; this radius was large enough that no reflection from this boundary could reach the bubble during the time frame of the analysis, so the remote (nonplane) boundaries of the model were left as noflow (rigid wall) boundaries. The plane symmetry boundaries at $\pm 1^{\circ}$ were also left as no-flow boundaries, as the flow in adjacent wedge segments would preclude flow across these boundaries.

Because of the large volume of surrounding fluid modeled for this problem, several different model regimes were defined. A number of different analyses were conducted at different stand-off 
distances from the boundary of interest; a typical finite element analysis model that was used is shown in Fig. 4. The overall geometry of this model is shown in Fig. 4(a); in this figure the vertical ( $y$ direction) line segment is the axisymmetric symmetry axis, and the narrow triangular area perpendicular to this axis is the constant pressure surface or rigid wall boundary. An expanded view of the area where these intersect [i.e., the lower left corner of Fig. 4(a)] is shown in Fig. 4(b). In this figure, the second triangular area above the bottom triangular area bisects the axis of the charge.

The finite element mesh used in this analysis is shown in Fig. 4(c), and consists of 30,183 Eulerian elements. Initially, 32 of these elements contain TNT, and the remainder contain seawater. Figure 4(d) shows an expanded view of the region near the charge [the lower left corner of Fig. 4(c)]. The lower boundary in this figure, parallel to the $x$ axis, is the constant pressure surface or rigid wall boundary of interest. For the analysis model shown in Fig. 4, the boundary of interest is located $97.5 \mathrm{~cm}$ below the initial center of the $20-\mathrm{cm}$ high, 20-cm diameter explosive charge.

\section{NUMERICAL RESULTS AND DISCUSSION}

The radius verus time behavior predicted for the explosion product gas bubble of the first problem [the free-field bubble at $178.6 \mathrm{~m}$, corresponding to one of Swift and Decius's (1950) experiments] is shown in Fig. 5. Also shown in this figure for comparison purposes are the experimentally determined first and second maximum bubble radius and period. Excellent agreement is seen between the experimental and finite element analysis results for the first maximum bubble radius and bubble period, indicating that the state equations, state equation parameters, and modeling assumptions (used for both the first and second problems) adequately model the underlying physics of the problem, up until the time of the first bubble minimum.

Also plotted in Fig. 5 is the radius versus time behavior predicted by Herring's (1950) approximate analytical solution to this problem, obtained by separating variables in the energy equation and numerically integrating the resulting expression:

$$
t=\left(\frac{3 \rho_{0}}{2 P_{0}}\right)^{1 / 2} \int_{r_{0}}^{r} \frac{d a}{\left[\left(r_{\max } / a\right)^{3}-1\right]^{1 / 2}}
$$

In this analysis, Herring assumed that the fluid surrounding the explosion gas bubble is incompressible and neglected the internal energy of the gas. For convenience, the maximum radius of the bubble is used as a substitute for the initial velocity of the bubble's bounding surface and is given the same value as the maximum radius from the finite element analysis. The initial radius of the bubble was taken as zero for simplicity. This curve was plotted to show that the shape of the radius versus time curve predicted by finite element analysis is in qualitative agreement with that from the analytical solution of the simplified problem.

One clear distinction is apparent between these two curves: there is a considerable energy loss between pulsations in the finite element analysis, as evidenced by the decreasing maximum radius and period of the bubble. This is due to acoustic radiation when the bubble is near minimum radius. As there is no such energy loss mechanism in the analytical solution, the pulsations for this solution are just mirror images of the first pulsation. Figure 6 shows the pressure, impulse, and fluid velocity predicted by finite element analysis at a point located at a distance equal to two maximum first bubble radii from the center of the bubble. The considerable impulse caused by the relatively low pressure but long duration bubble pulse emitted near the first bubble minimum is clearly seen in this plot. As the relatively long period of time between the impact of the initial shock wave and the secondary bubble pulse may cause the secondary pressure pulse to reinforce the bending mode vibration initiated in a target marine structure by the initial shock wave, this secondary pressure pulse could potentially cause significant damage to a target.

The experiment conducted by Swift and Decius (1950) also measured the second bubble maximum radius and oscillation period, and the measured values were even smaller than those predicted by our finite element analysis as shown in Fig. 5. This indicates that an energy loss mechanism, in addition to acoustic radiation appears to be important in this case. A likely explanation for this was given by Hicks (1970), who noted that in photographs taken of deep (nonmigrating) explosion gas bubbles, the bubble is unstable near its minimum radius and numerous needlelike fluid jets penetrate into the gas bubble; this fluid spray can then significantly cool the hot bubble gases. Cole (1948) provided photographs illustrating this phenomena. This phenomena is not seen when the bubble is migrating, e.g., when it is near a boundary. 


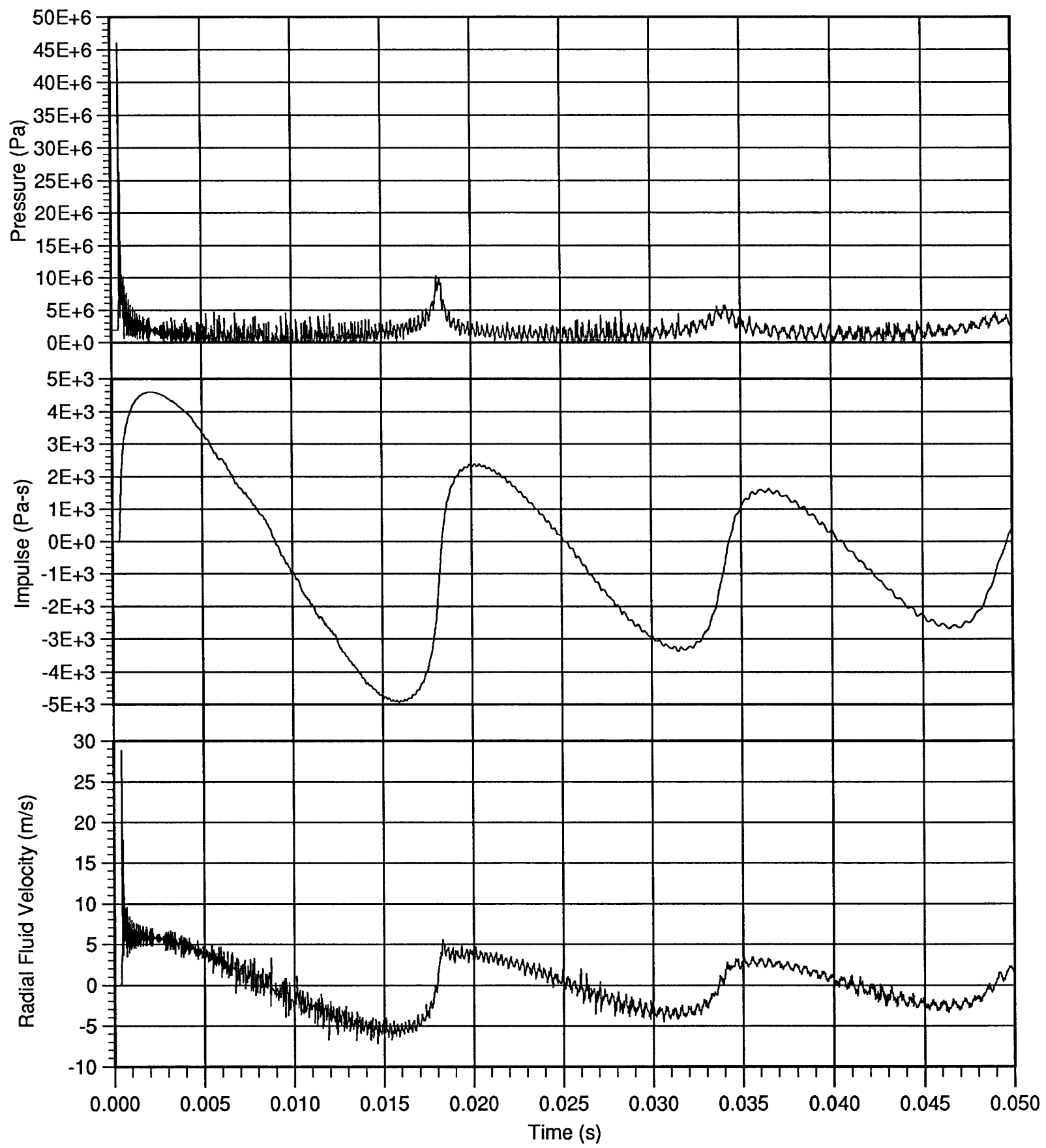

FIGURE 6 Pressure, impulse, and fluid velocity at a point two maximum free-field radii from the charge center for the spherical bubble problem.

The close agreement between Swift and Decius' (1950) experimental results and our finite element analysis results indicates that our state equations, methods, and assumptions seem to be adequate at least up to the time of the first bubble minimum. Beyond that time, they may or may not be accurate for cases in which there is a boundary near the bubble. Analyses for the bubble near a simple boundary problem was thus restricted to the first bubble pulsation.

To assist in quantification of analysis results for the bubble near a simple boundary problem, a free-field (no nearby boundary) analysis was conducted using an axisymmetric, half-symmetry 
model. In this 23,670 element model, all elements below the plane bisecting the charge axis were eliminated. This symmetry plane was then made a no-flow boundary. This analysis showed that the bubble resulting from detonation of the cylindrical charge became very nearly spherical shortly after detonation and remained so throughout the transient analysis. A maximum bubble radius of 70.65 $\mathrm{cm}$ was predicted, and the minimum radius of 22.32 $\mathrm{cm}$ occurred at $14.47 \mathrm{~ms}$ after detonation. These

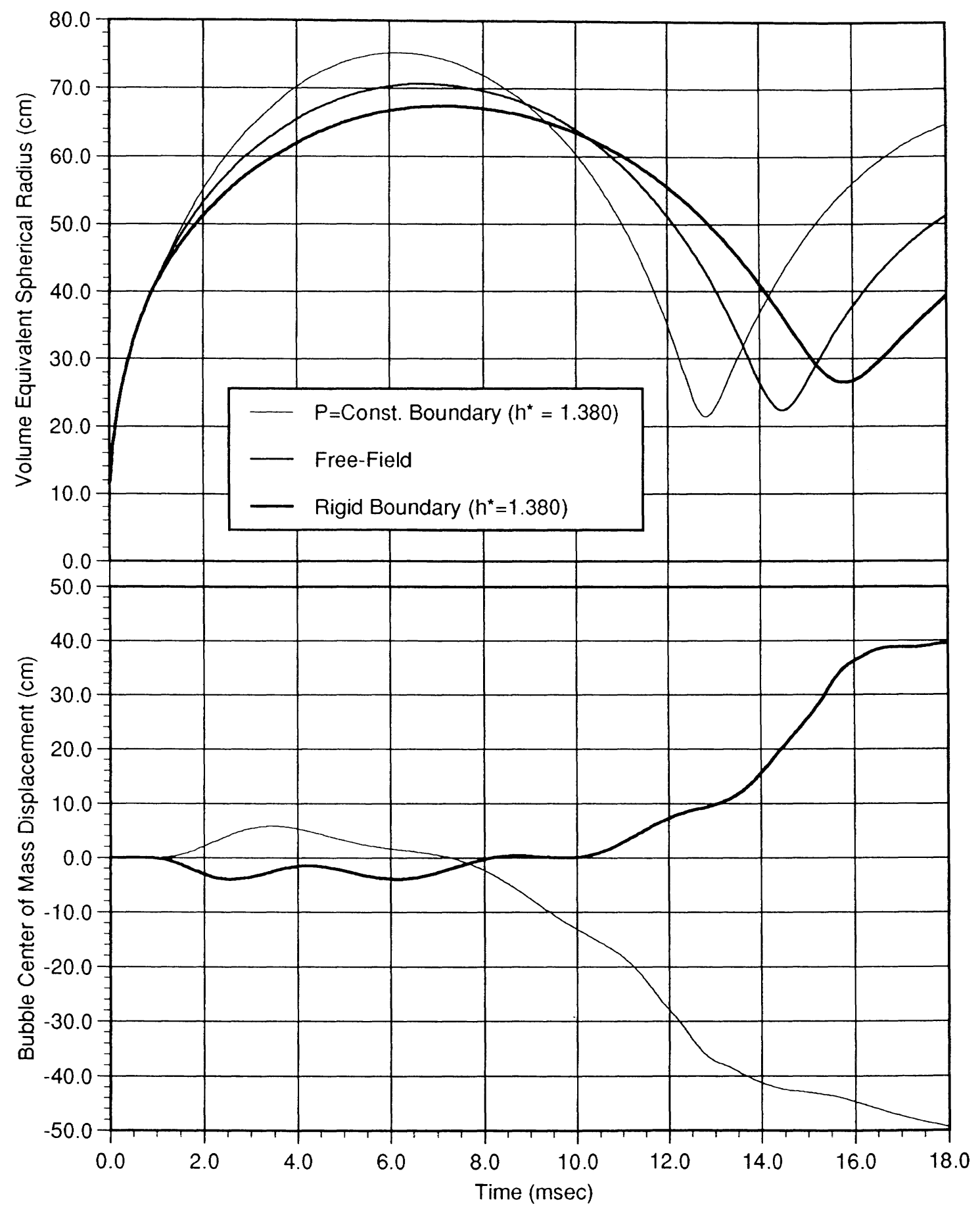

FIGURE 7 Equivalent spherical radius and center of mass displacement versus time behavior for the bubble near the boundary problem (typical). 


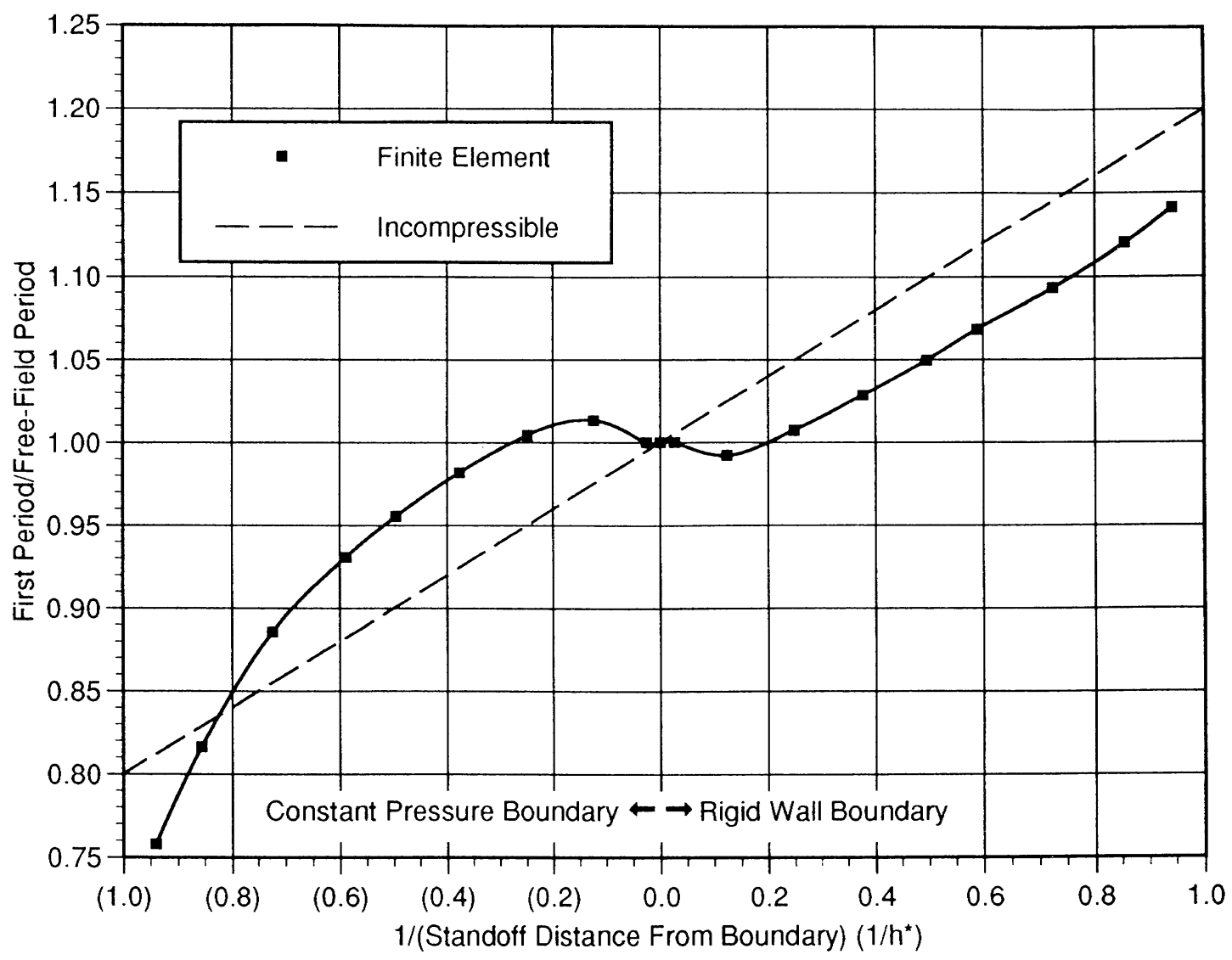

FIGURE 8 Boundary surface effect on the first bubble period versus the inverse stand-off distance from the boundary (nondimensionalized) for the bubble near the boundary problem.

quantities were used for comparison with analyses in which a rigid wall or constant pressure surface was present.

The dimensionless parameter $h^{*}$ was defined as the stand-off distance between the center of the charge and the nearest point on the plane constant pressure or rigid wall boundary, in units of maximum free-field radii. Analyses were conducted at stand-off distances of $h^{*}=1.062,1.168,1.380$, $1.698,2.017,2.654,4.034$, and 8.068 ; the typical model shown in Fig. 4 is for $h^{*}=1.380$. The number of Eulerian elements used in these models ranged from 28,680 for $h^{*}=1.062$, to 50,368 for $h^{*}=8.068$. Using the peak wave velocity seen in the fluid during these analyses together with the free-field period, it was determined that a plane constant pressure or rigid wall boundary could have no effect beyond an $h^{*}$ value of 40 during the first expansion-contraction cycle.

Typical finite element analysis results for bubble "volume equivalent spherical radius" (the radius of a spherical bubble with the same volume as the actual bubble) and center of mass displacement time histories are illustrated in Fig. 7. The cases shown in this figure are for a constant pressure surface and a rigid wall located at 1.380 maximum free-field radii from the center of the charge. The free-field radius versus time behavior is also shown in this figure. In this and subsequent graphs, displacements are considered to be positive if they are toward the boundary and negative if away from the boundary. Figure 7 shows the significant impact these boundaries can have on the radius, period, and migration of the bubble. In addition to the expected initial migration away from and subsequent migration toward the rigid boundary (and the opposite for the constant pressure boundary), the effect of the finite wave speed in a compressible media can be seen in the displacement versus time curve. There was no displacement until the initial shock wave reencountered the bubble after reflecting off the boundary (with a sign change at a constant pressure boundary). 
Finite element analysis results for the variation of the first bubble period with stand-off distance to the two types of boundaries modeled are given in Fig. 8. For convenience, the abscissa coordinates in this figure are the inverses of the stand-off distances nondimensionalized by the maximum freefield radius, i.e., $1 / h^{*}$; the ordinate coordinates are the bubble periods nondimensionalized by the free-field period. Also plotted in Fig. 8 for comparison purposes is the nondimensional bubble period $\left(T^{*}\right)$ versus the nondimensional inverse stand-off distance relationship predicted from an approximate analysis by Herring (1950). In Herring's analysis, the fluid is assumed to be incompressible, the gas bubble is assumed to have negligible internal energy and to remain spherical, and terms of order $\left(1 / h^{* 2}\right)$ and higher are neglected. Herring's formula for the bubble period is

$$
T^{\prime}=T\left(1 \pm \frac{r_{\mathrm{ave}}}{4 h}\right)
$$

where $T^{\prime}$ is the modified period in the presence of a boundary, $T$ is the free-field period, $r_{\text {ave }}$ is the average bubble radius over oscillation, $h$ is the stand-off distance to the boundary, and the upper sign is for a rigid wall, the lower for a constant pressure boundary.

Our finite element nondimensional period versus nondimensional inverse stand-off distance $\left(T^{*}\right.$ versus $1 / h^{*}$ ) curve has about the same slope as

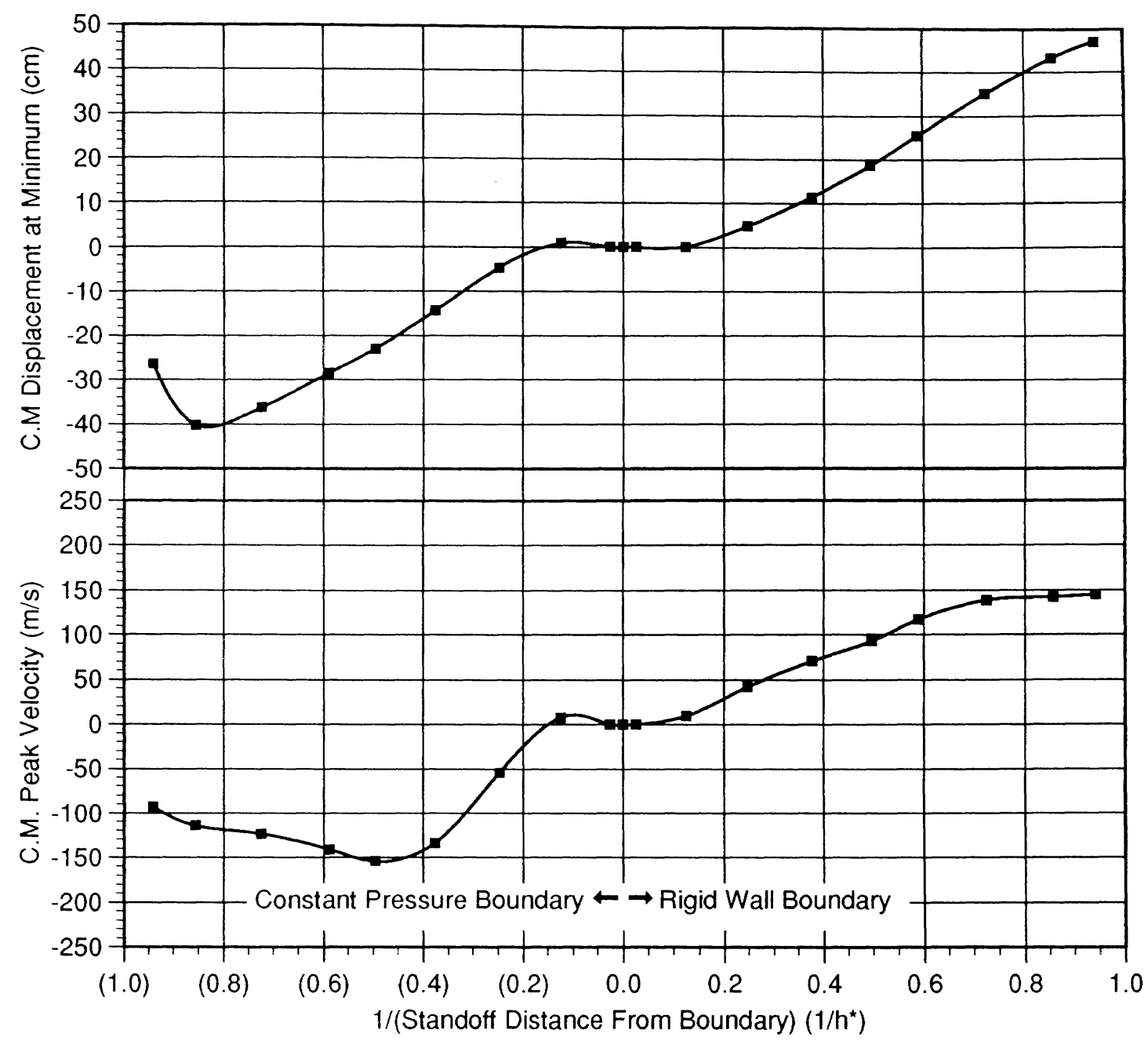

FIGURE 9 Bubble displacement at the first minimum and peak velocity versus the inverse stand-off distance from the boundary (nondimensionalized) for the bubble near the boundary problem. 


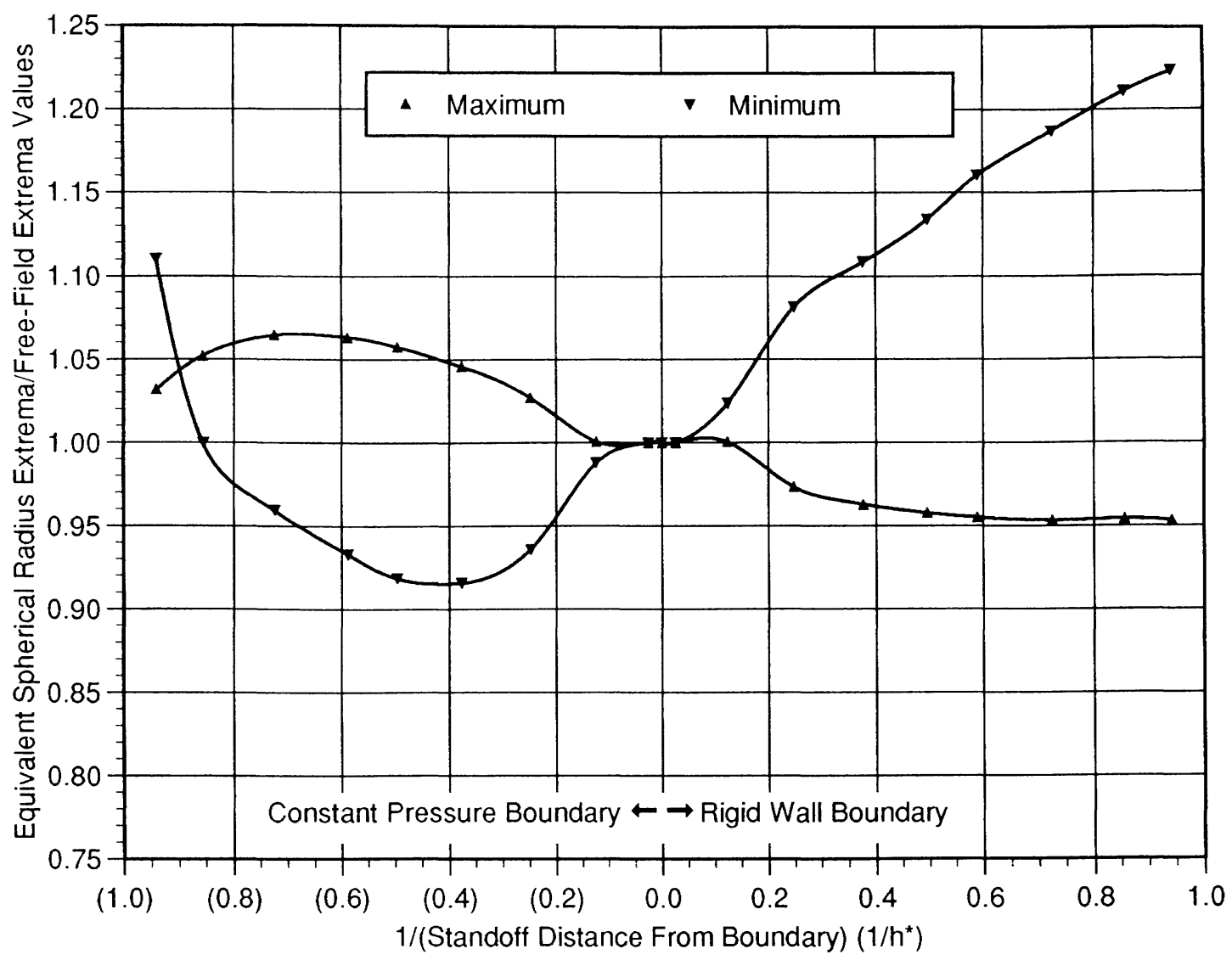

FIGURE 10 Equivalent spherical radius extrema versus the inverse stand-off distance to the boundary (nondimensionalized) for the bubble near the boundary problem.

the curve predicted by Herring's incompressible analysis over much of the stand-off range of interest. However, because of the finite wave speed in the real, compressible fluid we know that for very remote boundaries $\left(h^{*}>40\right)$ the boundary can have no effect on the first period of the bubble. Thus, not only must the real $T^{*}$ versus $1 / h^{*}$ curve leave the point where $1 / h^{*}$ equals zero with a zero slope, there must be a finite range of $1 / h^{*}$ values for which the slope of this curve is zero.

The fact that the linear part of the constant pressure surface part of this curve is offset further from Herring's prediction than the linear part of the rigid wall part of the curve, is probably due, at least in part, to the initial migration of the bubble. For the typical case shown in Fig. 7 when the bubble begins its collapse (i.e., it is at the "stationary point" where its volume is at a maximum), it is somewhat closer to the plane boundary surface for the constant pressure boundary case than for the rigid wall boundary case. The nondimensional period plotted in Fig. 8 is plotted as a function of the nondimensional initial inverse stand-off distance, not the inverse stand-off distance at the time the bubble reaches its maximum volume.

The displacement of the bubble center of mass at the time of the first minimum and the peak velocity of the bubble center of mass are plotted as a function of the inverse stand-off distance, nondimensionalized, in Fig. 9. This figure shows that the bubble displacement at the time of the first minimum can be an appreciable fraction of the initial stand-off distance for charges fairly near a boundary; because the secondary pressure pulse occurs near this time, this can significantly affect the damage done to nearby structures. Also of interest is the fact that the peak bubble migration velocities predicted by the finite element analysis incorporating compressibility are relatively modest.

Figure 10 shows the nondimensional equivalent spherical radius extreme (maximum and minimum) as a function of the nondimensional inverse stand-off distance. As with many of the other figures, a general "antisymmetry" about the free- 
field ordinate and abscissa axes is seen for detonations not too near a boundary surface. For closer detonations, the constant pressure boundary appears to have a different affect upon the extrema than the rigid wall boundary.

When comparing the rigid wall results against the constant pressure surface results plotted in Figs. 8, 9, and 10, several different "regimes" become apparent. For $1 / h^{*}$ smaller than about 0.35 , all of the results are generally antisymmetric. Between values of $1 / h^{*}$ of about 0.35 and 0.65 , the period, maximum equivalent radius, and center of mass displacement results are still roughly antisymmetric, but the minimum equivalent radius and peak velocity results are not. And at $1 / h^{*}$ values beyond about 0.85 a further change is seen in the displacement, peak velocity, and minimum equivalent radius curves. The differences for boundaries closer than $1 / h^{*}$ values of greater than
0.85 appear to be due primarily to bubble venting at the constant pressure surface caused by the increase in bubble radius due to the constant pressure surface combined with initial bubble migration toward the boundary. The differences seen at $1 / h^{*}$ values of between 0.35 and 0.65 appear to be related to bubble migration.

A plot of the bubble shapes predicted by our finite element analysis for the case in which the bubble is initially at $h^{*}=1.380\left(1 / h^{*}\right.$ about 0.725$)$ from a constant pressure surface is shown in Fig. 11. At the beginning of collapse the bubble appears to be nearly spherical, while in the final collapse stages it assumes a "sphere-cap" shape.

The final collapse stage shapes predicted by our analysis when the bubble is initially at $h^{*}=1.380$ from a rigid wall are shown in Fig. 12 (the bubble remains nearly spherical until this late collapse phase). The bubble shapes in Fig. 12 are much

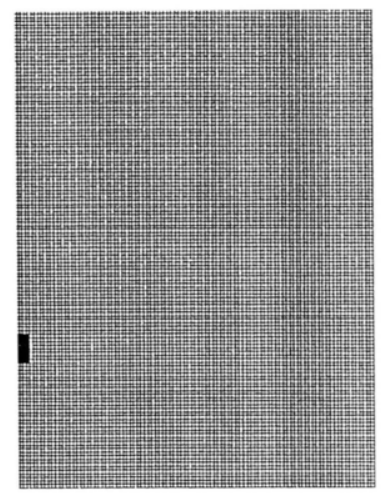

$t=0.00 T$

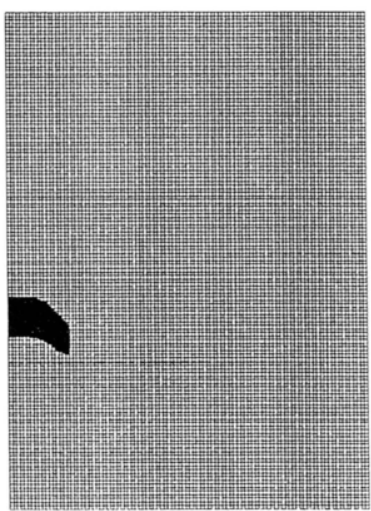

$t=0.95 \mathrm{~T}$

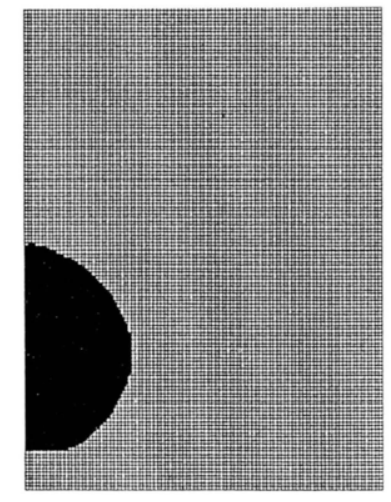

$t=0.50 \mathrm{~T}$

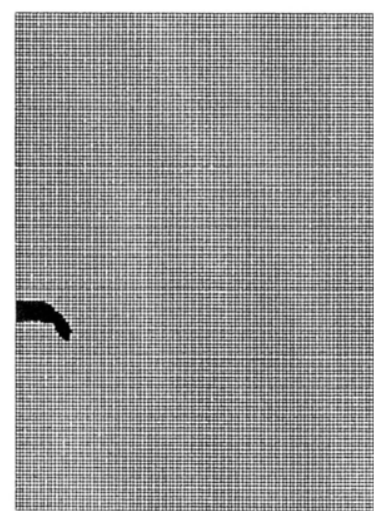

$t=0.98 \mathrm{~T}$

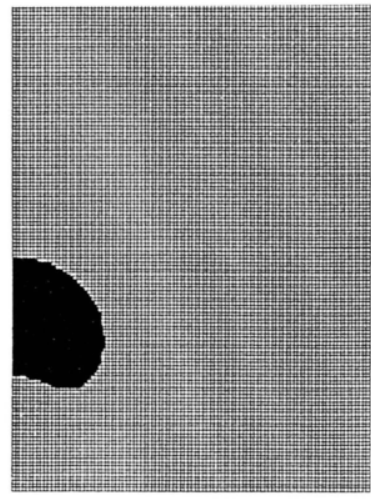

$t=0.80 \mathrm{~T}$

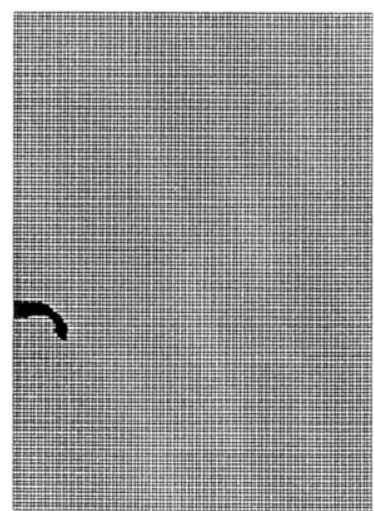

$t=0.99 \mathrm{~T}$

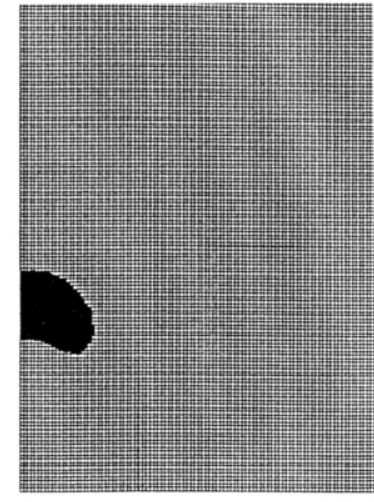

$t=0.90 \mathrm{~T}$

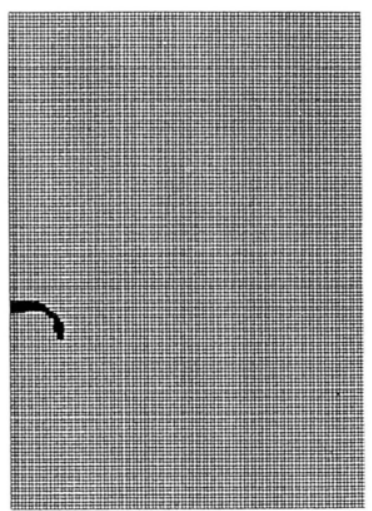

$t=1.00 \mathrm{~T}$

FIGURE 11 Shapes of the bubble for expansion and collapse at $h^{*}=1.380$ from a constant pressure boundary for the bubble near the boundary problem (boundary located at the bottom). 


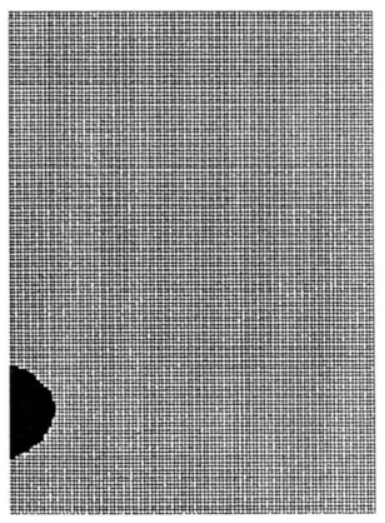

$t=0.95 \mathrm{~T}$

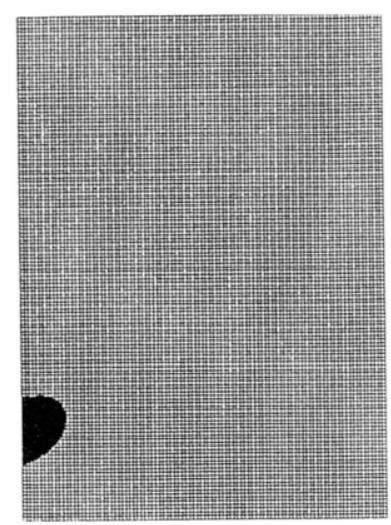

$t=0.98 \mathrm{~T}$

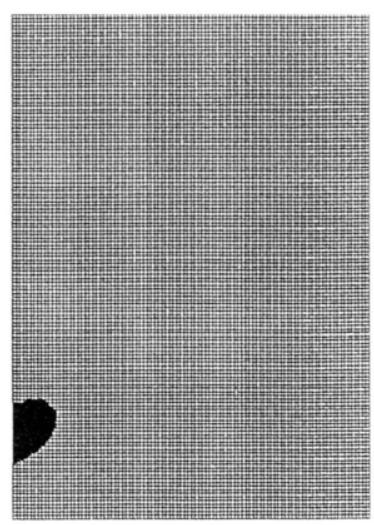

$\mathrm{t}=0.99 \mathrm{~T}$

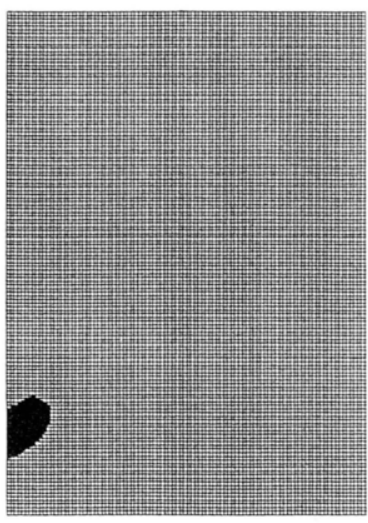

$\mathbf{t}=1.00 \mathrm{~T}$

FIGURE 12 Bubble shapes during the final collapse of the bubble at $h^{*}=1.380$ from a rigid wall for the bubble near the boundary problem (boundary located at the bottom).

closer to a "kidney" shape than a sphere cap. As with the results discussed above, the differences may be largely due to changes in the "effective stand-off distance" (due to changes in the bubble's maximum equivalent radius and the bubble's position at the beginning of the collapse phase). Our analysis of different rigid wall boundary cases (Chisum and Shin, 1995) has shown that the bubbles remain nearly spherical at $h^{*}$ values of greater than about 2.0, but bubble shapes are sensitive to stand-off distance at smaller stand-off distance values.

\section{CONCLUSION}

The application of multimaterial Eulerian analysis to investigation of explosion gas bubble phenomena has been demonstrated in this investigation of the effects of simple boundaries on explosion gas bubbles. The ability of this approach to yield very good results up until the first bubble minimum has been demonstrated; but beyond this time a more sophisticated approach that accounts for heat transfer may be needed, particularly if the boundary is so remote from the bubble that little migration occurs.

The boundary types chosen for this investigation are admittedly simple approximations to the types of boundaries of interest in the investigation of underwater explosions. However, they serve as a limiting case for many cases of practical interest: e.g., an explosion near a rigid but curved marine hull; an explosion near the ocean bottom or sur- face; or an explosion near a gas-filled bottle, tank, or compartment.

The finite element program MSC/DYTRAN used in this investigation was developed for conducting coupled Lagrangian-multimaterial Eulerian analysis of fluid-structure interaction problems. We have not used this capability in these completely Eulerian analyses, but the extension of this investigation to cases with a deformable Lagrangian boundary is not difficult.

The results discussed in this article are intended to demonstrate the ability to characterize the effects of very simple boundaries on a particular explosion gas bubble. Work is in progress to extend and refine the procedures and results from this investigation to more realistic problems, including deformable boundaries, a realistic airwater interface, and problems incorporating viscosity. The eventual goal is to provide accurate "semiempirical" equations for bubble parameters of interest sufficient to accurately determine the response of nearby marine structures to loading from any explosion gas bubble.

\section{REFERENCES}

Chisum, J. E., and Shin,Y.S., 1995, "Multimaterial Eulerian and Coupled Lagrangian-Eulerian Finite Element Analysis of Underwater Shock Problems," Report NPS-ME-95-001, Naval Postgraduate School, Monterey,CA.

Cole, R. H., 1948, Underwater Explosions, Princeton University Press, Princeton, NJ.

Dobratz, B. M., 1981, LLNL Explosives Handbook, 
Lawrence Livermore Laboratory, University of California, Livermore, CA.

Hartmann, G. K., and Hill, E. G., Editors, 1950, Underwater Explosion Research: A Compendium of British and American Reports, Office of Naval Research, Department of the Navy, Washington, DC.

Herring, C., 1950, "Theory of the Pulsations of the Gas Bubble Produced by an Underwater Explosion," in Underwater Explosion Research: A Compendium of British and American Reports, Vol. 2, Office of Naval Research, Department of the Navy, Washington, DC, pp. 35-130.
Hicks, A. N., 1970, "Effects of Bubble Migration on Explosion-Induced Whipping of Ships," NSRDC Report 3301.

MacNeal-Schwendler Corporation, 1995, MSC/DYTRAN User's Manual, Version 2.3, Los Angeles, CA.

Swift, E., and Decius, J. C., 1950, "Measurements of Bubble Phemomena III: Radius and Period Studies," in Underwater Explosion Research: A Compendium of British and American Reports, Vol. 2, Office of Naval Research, Department of the Navy, Washington, DC, pp. 553-599. 

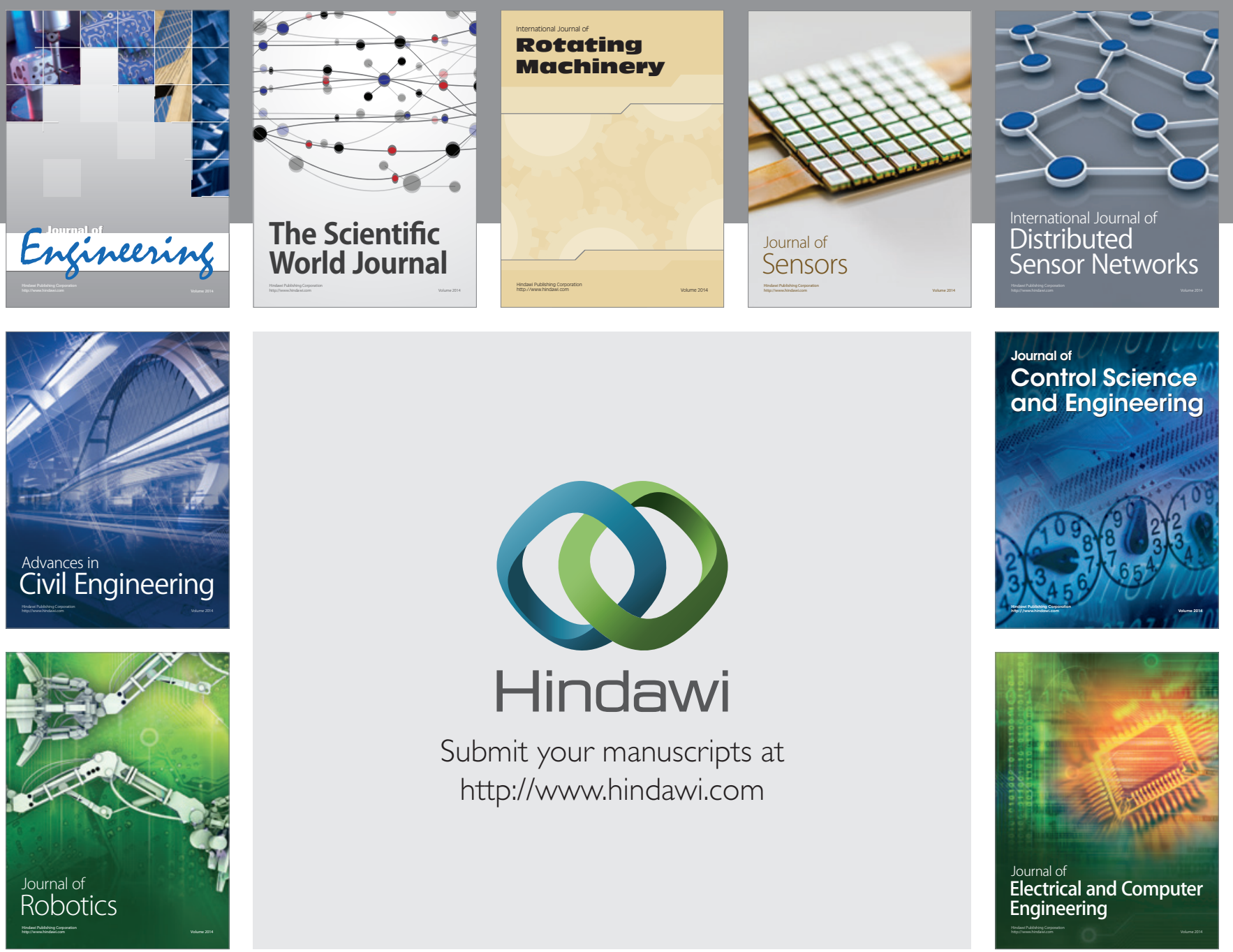

Submit your manuscripts at

http://www.hindawi.com
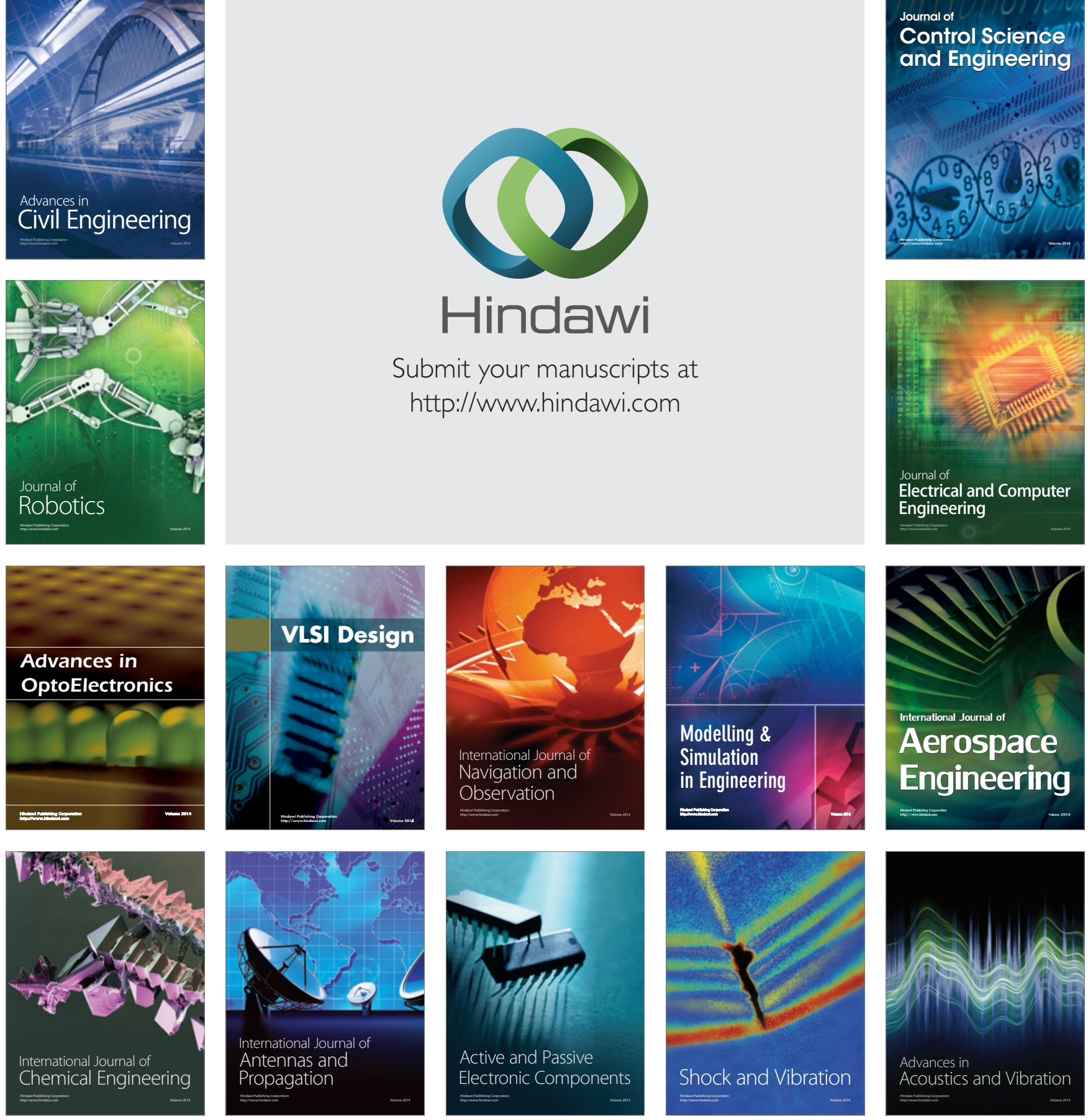